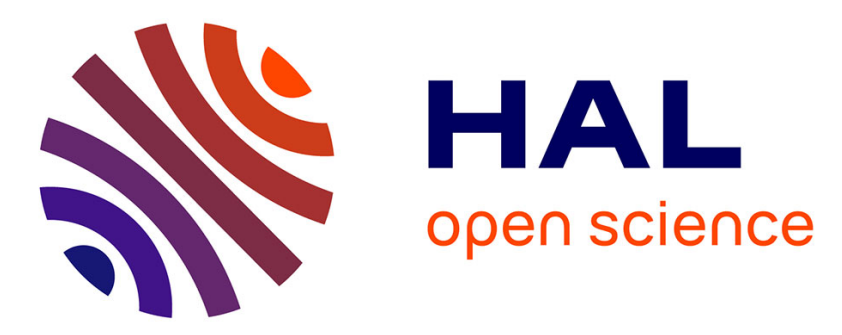

\title{
Alpha-stable interference modeling and Cauchy receiver for an IR-UWB ad hoc network
}

Hamza El Ghannudi, Laurent Clavier, Nourddine Azzaoui, François Septier, Paul-Alain Rolland

\section{- To cite this version:}

Hamza El Ghannudi, Laurent Clavier, Nourddine Azzaoui, François Septier, Paul-Alain Rolland. Alpha-stable interference modeling and Cauchy receiver for an IR-UWB ad hoc network. IEEE Transactions on Communications, 2010, 58 (6), pp.1748 - 1757. 10.1109/TCOMM.2010.06.090074 . hal-00685924

\section{HAL Id: hal-00685924 https://hal-imt.archives-ouvertes.fr/hal-00685924}

Submitted on 6 Apr 2012

HAL is a multi-disciplinary open access archive for the deposit and dissemination of scientific research documents, whether they are published or not. The documents may come from teaching and research institutions in France or abroad, or from public or private research centers.
L'archive ouverte pluridisciplinaire $\mathbf{H A L}$, est destinée au dépôt et à la diffusion de documents scientifiques de niveau recherche, publiés ou non, émanant des établissements d'enseignement et de recherche français ou étrangers, des laboratoires publics ou privés. 


\title{
$\alpha$-stable Interference Modeling and Cauchy Receiver for an IR-UWB ad hoc Network
}

\author{
Hamza El Ghannudi, Laurent Clavier, Member, IEEE, Nourddine Azzaoui, \\ François Septier Member, IEEE, and Paul-Alain Rolland
}

\begin{abstract}
In this paper we investigate ad hoc networks based on impulse radio ultra wideband. Due to multiple access, the interference distribution is not Gaussian. One important reason for errors is the presence of close interferers generating pulse collision. However such events are rare and we propose an $\alpha$-stable model compatible with this fact due to its heavy tailed distribution. We derive the analytical expression of the two significant parameters. They depend on the attenuation coefficient, the users' density, the pulse collision probability and the pulse shape. We finally propose receiver strategies (Cauchy receiver and $p$-norm) that outperforms the classical Gaussian receiver.
\end{abstract}

Index Terms-Multiple Access Interference (MAI), UWB, ad hoc networks, $\alpha$-stable distribution.

\section{INTRODUCTION}

In dense ad hoc networks, multiple access is the main component of interference. In other contexts and systems, it is considered to be the sum of numerous independent and identically distributed random variables. As a consequence, a Gaussian approximation is used and often gives accurate results. However, the ad hoc configuration modifies the MAI distribution: the interfering pulses amplitude may importantly vary and the MAI is conditioned by the presence of strong interferers [1]-[3]. Sousa shows in [4] that it can be modeled as an $\alpha$-stable random variable. This result is also presented with lognormal shadowing and Rayleigh fast fading in [5]. In [6], Pinto derives an exact expression for the error performance of a narrowband communication system subject to multiple UWB interferers and additive white Gaussian noise. A framework where the UWB interferers are spatially scattered according to an infinite Poisson field and are operating asynchronously is introduced. A symmetric stable distribution to model the UWB

Paper approved by D. I. Kim, the Editor for Spread Spectrum Transmission and Access of the IEEE Communications Society. Manuscript received February 4, 2009; revised October 23, 2009.

M. El Ghannudi is with Department of Electronic and Information Engineering (DIEI), University of Perugia, Italy. (e-mail: hamza.ghannudi@diei.unipg.it).

M. Clavier and P.-A. Rolland are with IEMN (Institute of Electronics, Microelectronics and Nanotechnology, UMR CNRS 8520) and IRCICA (Research Institute on software and hardware components of the future for information and communication, FR CNRS 3024) in Lille, France. (e-mail: laurent.clavier@telecom-lille1.eu, paul-alain.rolland@iemn.univ-lille1.fr).

M. Clavier and F. Septier are with Institut TELECOM, TELECOM Lille 1. M. Septier is also with the "Laboratoire d'Automatique, Génie Informatique et Signal" (LAGIS UMR CNRS 8146). (e-mail: francois.septier@telecomlille1.eu).

N. Azzaoui is with the systems modeling and safety laboratory (LM2S), Charles Delaunay Institute (ICD), FRE CNRS 2848, UTT, France. (e-mail: Nourddine.azzaoui@utt.fr).

Digital Object Identifier 10.1109/TCOMM.2010.090074 interference at the output of a linear narrowband receiver is used. Win et al. have recently proposed a mathematical framework for network interference in [7]. They show that, when interferers are scattered according to a Poisson process, the aggregate interference amplitude follows a symmetric stable distribution.

Similarly for the proposed physical layer, it is shown in [8] that the Gaussian approximation significantly underestimates the Bit Error Rate (BER) of practical Time Hopping (TH) Pulse Position Modulation (PPM) systems. Its validity increases with the number of interferers [1] but do not adequately predict the BER at low values of the user bit rate and of the pulse repetition frequency [2]. A way to mitigate the effect of the MAI by optimizing the TH codes in asynchronous $\mathrm{TH}$ is proposed in [9] but only works because interference is not Gaussian. To estimate the BER of an IR-UWB multiuser transmission, several other methods have been proposed: in [10] a composite method is used and in [11] a method based on the characteristic function; in [12], the calculation is based on the fact that interference is due to collisions occurring between pulses belonging to different transmissions.

We suggest in this paper that the ad hoc configuration gives the shape of the MAI distribution which is then accurately represented by symmetric $\alpha$-stable distributions. Stable laws can be seen as an extension of the Gaussian laws but with heavier tails so that they are better suited for rare events modeling. Two parameters have to be determined and are obtained from the channel attenuation and the physical layer definition. In a first step, we develop an analytical work based on a single path channel. One intended application is for up-converted UWB signals in the $60 \mathrm{GHz}$ band with sensors using sectorized antennas [13], [14]. In such a context, the multipath influence is low and, especially in an ad hoc scenario with rather short links, the reflected paths only carry little energy compared to the direct one [15]. The proposed theoretical approach is then well adapted. We derive the analytical expression of the two parameters and show the validity of the model.

We then go one step further to validate our approach and consider the residential IEEE 802.15.4a UWB channel model. Time-hopping impulse radio with a simple multipleaccess scheme like an ALOHA protocol, is suitable for sensor networks design [16], [17]. We propose different receiver schemes that take into account the stable noise. We show that a Cauchy receiver (although it is not generally optimal) or 


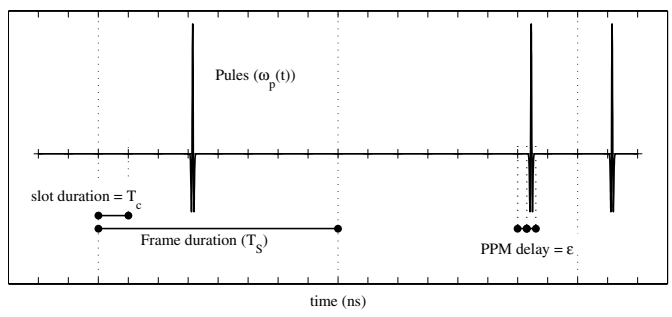

Fig. 1. Illustration of some system parameters.

the use of the p-norm (as proposed in [18] for a generalized Gaussian interference model) significantly improve the system performance although the important multipath tend to reduce the benefit. However, the classical Gaussian receiver is outperformed and we show $(a)$ that our model is adequate for MAI and $(b)$ that taking this fact into account allows a better design of the receivers.

The rest of the paper is organized as follows: in section II we present the network configuration and the system model. In section III, with a single path channel and ignoring the near field in the attenuation calculation (this means that when the transmitter receiver distance gets close to zero, the receive power increases to infinity; although unrealistic, this assumption has no significant impact on our conclusions), we show that the MAI is an $\alpha$-stable random variable and derive the parameters' expressions. Finally, in section IV, we propose receiver architectures adapted to the proposed interference model and evaluate its performance in Gaussian and multipath channels.

\section{NETWORK CONFIGURATION AND SYSTEM MODEL}

An ad hoc network is a self-configuring network of mobile devices connected by wireless links. We consider throughout this paper a MAC layer based on an Aloha protocol that can have close performance to a CSMA protocol as shown in [16] for a multihop mobile wireless network. This means that no special effort is made to prevent simultaneous neighboring transmissions from occurring.

We consider a receiver located at the center of a circle $C$ of radius $R$. The desired user (who will be denoted by index 0 in the following) is at a given distance $d_{u}$ from the receiver and interferers are uniformly distributed in $C$. Let $N$ represent the number of interferers in the area. It depends on the users' density, the maximum range for a signal to be received and the traffic in the network.

Asynchronous TH-PPM-UWB with binary data is considered [19] and represented in Figure 1. Let $\omega(t)$ be the transmitted signal corresponding to one source bit. It has a normalized energy equal to 1 and it is the repetition of $N_{S}$ basic pulses $\omega_{p}(t)$ :

$$
\omega(t)=\sum_{j=1}^{N_{S}} \omega_{p}\left(t-j T_{S}-c_{j}^{(k)} T_{c}\right)
$$

where $T_{S}$ is the frame duration, $c_{j}^{(k)}$ is the $j^{\text {th }}$ coefficient of the pseudo-random $\mathrm{TH}$ code and $T_{c}$ is the slot duration. The transmitted signal for user $k$ is then given by:

$$
S^{(k)}(t)=\sum_{i=-\infty}^{+\infty} \sqrt{E^{(k)}} \omega\left(t-i N_{S} T_{S}-a_{i}^{(k)} \epsilon-\delta^{(k)}\right),
$$

where $E^{(k)}$ is the transmitted energy for each pulse, $a_{j}^{(k)}$ is a binary data value, $\epsilon$ is the basic shift introduced by PPM and $\delta^{(k)}$ the delay between user $k$ and user 0 .

We adopt a single user correlation receiver where the received signal is correlated with a template waveform $m(t)=$ $\omega(t)-\omega(t-\epsilon)$ after a perfect time synchronization on the main path. Without loss of generality, we consider the detection of the source bit $a_{0}^{(0)}$. The receiver output $Z_{d}$ is:

$$
Z_{d}=\int_{0}^{N_{S} T_{S}} \sum_{k=0}^{N}\left(S^{(k)}(t) * h^{(k)}(t)+n(t)\right) m(t) d t,
$$

where $*$ stands for convolution, $h^{(k)}$ is the channel impulse response and $n(t)$ is the thermal noise, circularly symmetric white and Gaussian.

Variable $Z_{d}$ can be expressed as the sum of four terms [20]: $Z_{d}=Z_{u}+Z_{M P I}+Z+Z_{n}$, where $Z_{u}, Z_{M P I}, Z$ and $Z_{n}$ are, respectively, the contributions of the desired signal, the multipath interference, the multiuser interference and the additive white Gaussian noise. If a Gaussian assumption is made for the global interference, the optimal decision is taken by comparing $Z_{d}$ with a zero-valued threshold. For independent and equiprobable transmitted bits, the average BER is $P_{e}=\mathbb{P}\left(Z_{d}<0 \mid a_{0}^{(0)}=0\right)$ where $\mathbb{P}(X)$ denotes the probability of event $X$.

In the next section, to compute the semi-analytical error probability, we neglect the multipath effect $\left(Z_{M P I}=0\right)$. Equation (3) then becomes:

$$
Z_{d}=\sum_{k=0}^{N} \gamma_{k} \int_{0}^{N_{S} T_{S}}\left(S^{(k)}\left(t-\tau_{k}\right)+n(t)\right) m(t) d t,
$$

where $\gamma_{k}$ is the channel attenuation and $\tau_{k}$ the propagation delay. In ad hoc networks, links are short and direct, which means that multipath carry little energy, especially in the millimeter wave band [15]. We present in Figure 2 the impact of the multipath in our considered scenario: the channel model [21] is obtained from a measurement campaign in a small room presented in [22]. Directive antennas $\left(100^{\circ}\right.$ at the emitter and $30^{\circ}$ at the receiver) are used to further reduce the multipath influence. Multipath have in this context a very limited impact. Consequently, in section III, we take a single path channel to derive the MAI distribution. However, we will also show in section IV that the proposed model is still appropriate in multipath channels and can lead to significant improvement of the receiver design.

Finally, we denote $X=Z_{u}+Z_{n} ; X$ is a Gaussian random variable with mean $Z_{u}$ and same variance as the Gaussian noise $Z_{n}$. Let $f_{X}(x)$ be its probability density function. The mean BER $P_{e}$ is:

$$
P_{e}=\int_{-\infty}^{+\infty} \mathbb{P}\left(Z<-x \mid a_{0}^{(0)}=0, Z_{n}\right) f_{X}(x) d x
$$




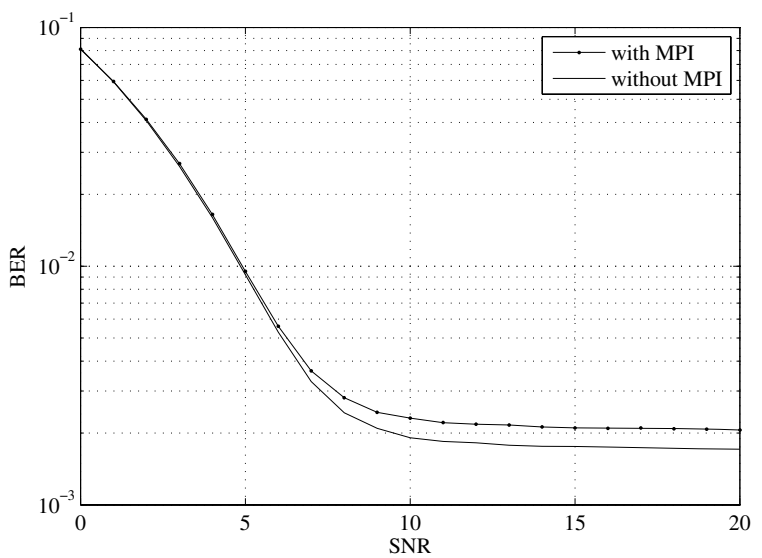

Fig. 2. Influence of multipaths in our $60 \mathrm{GHz}$ line of sight scenario. There is a mean of 35 objects in a circle of radius $R=5 \mathrm{~m}$.

\section{Modeling MUltiple ACCess Interference}

\section{A. General idea}

At the receiver, we develop (3) with single path channels. We only consider pulses that effectively interfere with the useful signal. Let $\kappa_{R}$ be the random variable representing their number. The MAI random variable $Z$ can then be written as:

$$
Z=\sum_{k=1}^{\kappa_{R}} \gamma_{k} \psi_{k}
$$

$$
\text { with } \quad \psi_{k}=\int_{0}^{T_{S}} \omega_{p}\left(t-\Delta_{k}\right)\left(\omega_{p}(t)-\omega_{p}(t-\epsilon)\right) d t,
$$

where $\Delta_{k}$ is the total delay between the interfering and the reference signals. The random variable $\Delta_{k}$ is uniformly distributed over $\left[-T_{S}, T_{S}\right]$ due to asynchronous transmission and independence between desired and interfering links. In (6), $\left(\psi_{k}\right)_{k=1, \cdots, N}$ are independent, identically distributed and bounded random variables. Their probability density function is even. The $\left(\gamma_{k}\right)_{k=1, \cdots, N}$ are positive, independent identically distributed random variables and determine the statistical properties of $Z$. Let now suppose that we are placed in a dense traffic: $\kappa_{R}$ is very large. Since $\left(\gamma_{k}\right)_{k=1, \cdots, \kappa_{R}}$ have finite variances, it is the same for the products $\left(\gamma_{k} \psi_{k}\right)_{k=1, \cdots, \kappa_{R}}$.

One intuitive approach, considering an asymptotic regime where the number of intereferers grows to infinity while the contribution of each interferer to the total MAI becomes infinitesimal, would be to use the central limit theorem. The MAI, then expressed as the normalized sum:

$$
Z^{\left(\kappa_{R}\right)}=\frac{1}{\sqrt{\kappa}_{R}} \sum_{k=1}^{\kappa_{R}} \gamma_{k} \psi_{k}
$$

converges in law to a normal distribution with the same variance as $\gamma_{k} \psi_{k}$. This means that $Z^{\left(\kappa_{R}\right)}$ is asymptotically Gaussian and by the sequel may be approximated with a normal distribution when $\kappa_{R}$ is large enough.

However, in the general case, the asymptotic regime is not easily reached. An in-depth study is proposed in [23] and only a large number of users, a large processing gain and a large number of repetitions lead to a Gaussian MAI. Besides, we can discuss the condition of finite variance on $\gamma_{k}$. If it can seem natural because it represents a channel attenuation, the interference is compared to the desired link attenuation $\gamma_{0}$. If this link is long but the interfering one is short, the observed relative values of $\gamma_{k}$ may be "very large". These rare events are very important in our context and give an impulsive nature to attenuations. To capture these situations, heavy tailed distributions with infinite variance can be well suited while models with finite second order will fail to do so. The generalized central limit theorem has then to be used (see [24, p. 22] or [25, p. 9]) and states that the MAI (for large $\kappa_{R}$ ) falls in the domain of attraction of a random variable with a stable distribution.

This infinite variance hypothesis is equivalent to neglecting the near field ${ }^{1}$ when calculating the amplitude attenuation of the signal $\gamma_{k}$. We suppose that it is given by $\gamma_{k}=d_{k}^{-a / 2}$, $a$ being the attenuation coefficient and $d_{k}$ the distance from interferer $k$. If interferers are uniformly distributed inside the circle $C$ of radius $R$, the attenuation probability density function is:

$$
f_{\gamma_{i}}(x)=\frac{4 x^{-\frac{4}{a}-1}}{a R^{2}} \quad \text { for } \quad R^{-\frac{a}{2}} \leq x<+\infty .
$$

Its variance is infinite for $a>2$. Even if this hypothesis does not correspond to reality, it is an accurate way to represent the high variability in $\gamma_{k}$ and the fact that there are many far users with small $\gamma_{k}$ but few close ones with high $\gamma_{k}$.

We have mainly focused here on the impact of ad hoc configuration on the MAI. Other reason due to impulse UWB are also unvalidating the Gaussian approximation: the fact that the sum is not normalized or that the number of interfering pulses is not always large and is better modeled with a discrete random variable. As it is usually considered in similar situations [4], [6], one can assume that $\kappa_{R}$ has a Poisson distribution. For a discussion about these considerations, an overview of proposed modeling solutions (Laplace, Generalized Gaussian, Cauchy, $\alpha$-stable, Middleton class A...) is proposed in [26] and we do not extend the discussion here. In this paper, using the theory of compounded Poisson processes we derive in section III-C that the random variable $Z$ can be modeled with an $\alpha$-stable law when the near field is neglected. Consequence of the ad hoc configuration, this result is valid for DS-CDMA and FH-CDMA [4] and extended in this paper to TH-PPM-UWB.

\section{B. The $\alpha$-stable random variables.}

Stable distributions are a rich class of probability distributions that include the Gaussian $(\alpha=2)$, Cauchy $(\alpha=1)$ and Lévy ( $\alpha=0.5)$ laws in a family that allows skewness and heavy tails. There are several ways to define stable distributions [25, chapter 1]. One concerns the stability property: the family of stable distributions is preserved under convolution. Another is that they approximate the distribution of normalized sums of independent and identically distributed random variables (generalized central limit theorem).

\footnotetext{
${ }^{1}$ If we do not neglect the near field, for $d$ less than a given distance $d_{0}$ the received power would not follow the same law as the one we use. A better model would be to then consider that $\gamma_{k}=d_{0}^{-a / 2} \forall d<d_{0}$.
} 
The class of stable distributions has provided useful models in many application fields (electrical engineering, physics, signal processing...) but they still have a limited impact in digital communication although they proved to be accurate for modeling impulsive noises [24, chapter 9]. Of course, other heavy tailed probability models are available but the stability property makes this class very attractive. Besides they are defined by four parameters (some more details can be found in appendix A), the characteristic exponent $\alpha$ being one of them: it measures the thickness of the tail of the distribution $(0<\alpha \leq 2)$. However, in the general case, no closed-form expression of the probability density function is available which makes more difficult the implementation of optimal signal processing solutions.

\section{The MAI log-characteristic function}

The MAI random variable has a symmetric $\alpha$-stable distribution if we can write its log-characteristic function as $\varphi_{Z}(\omega)=-\sigma|\omega|^{\alpha}$. For the theoretical framework we consider in a first step that interferers are in a circle of radius $R$, the receiver being the center and we make $R$ tends towards infinity. We assume that the number $N$ of active interfering links follows a Poisson law of parameter $\frac{\bar{N}}{\pi R^{2}}$ which represents the mean number of interferers per unit area, $\bar{N}$ being the mean number of interferers. For a given $N$, the conditional law of the number $\kappa_{R}$ of interfering pulses coming from inside the circle $C$ is binomial $B(N, q)$, where $q$ is the probability that one interfering pulse falls into the integration interval at the receiver. Due to the uniform distribution of the delays $\Delta_{k}$ between users, $q=\frac{3 T_{m}}{T_{s}}$ where $T_{m}$ is the pulse duration and $3 T_{m}$ represents the duration of the vulnerability interval (a pulse falling in this interval will effectively create interference). Finally, a binomial law conditional to a Poisson law results in a Poisson law and its parameter is $\frac{\bar{N} q}{\pi R^{2}}$.

The MAI characteristic function is:

$$
\phi_{Z}(\omega)=\mathbb{E}\left[e^{j \omega\left(\sum_{k=1}^{\kappa} \gamma_{k} \psi_{k}\right)}\right],
$$

where $\kappa$ is the number of interferers in the circle with radius $+\infty$. Neglecting the near field for reasons explained in section III-A, we can show (see annex B) that the log-characteristic function can be written:

$$
\begin{aligned}
\varphi_{Z}(\omega) & =\frac{\bar{N} q}{R^{2}}|\omega|^{\frac{4}{a}} \int_{0}^{+\infty} \frac{d \phi_{\psi}}{d u}(u) u^{-\frac{4}{a}} d u \\
& =\frac{\bar{N} q}{R^{2}}|\omega|^{\frac{4}{a}} F,
\end{aligned}
$$

where $\phi_{\psi}(\omega)$ is the characteristic function of $\psi(\omega)$ (defined in (6)). The value $F$ is independent of $\omega$ so that $Z$ is a symmetric $\alpha$-stable random variable with parameters $\alpha=4 / a$ and $\sigma=$ $-\left(\bar{N} q / R^{2}\right) F$ (the two remaining parameters are zero). Since $\psi$ has finite moments, the integral to calculate $F$ exists when $a$ is larger than 2, which is the case in most of the situations. This result validates the $\alpha$-stable assumption. The main unrealistic hypothesis was to neglect the near field but Sousa showed in [4] that this aspect has a low impact.

\section{Model validity}

Channel attenuation is based on hypothesis from section III-A: $\gamma_{i} \propto d^{-a / 2}$ and we take $N_{S}=1$. A summary of parameters used in every simulation is presented in table I.

TABLE I

IMPULSE RADIO SYSTEM PARAMETERS USED IN SIMULATION.

\begin{tabular}{|c|c|}
\hline Parameter & Value \\
\hline Frame duration $T_{s}$ & $10 \mathrm{~ns}$ \\
\hline Pulse duration $T_{m}$ & $0.3 \mathrm{~ns}$ \\
\hline PPM delay $\epsilon$ & $0.3 \mathrm{~ns}$ \\
\hline$d_{u}$ & $1 \mathrm{~m}$ \\
\hline
\end{tabular}

To evaluate $\sigma$, we need to calculate $F$ in (9). It can be analytically obtained when $\omega_{p}(t)$ is a rectangular pulse that is why the comparison between simulations and theory is made with this pulse shape (although it is not a practical one). The characteristic function of $\psi_{k}$ (see (6)) is $\phi_{\psi}(\omega)=\frac{\sin (\omega)}{\omega}$. Then $F$ is given by:

$$
F=\frac{2^{-1-\frac{4}{a}} \sqrt{\pi} \Gamma\left(\frac{-2}{a}\right)}{\Gamma\left(\frac{1}{2}+\frac{2}{a}\right)}-\frac{2^{-2-\frac{4}{a}} \sqrt{\pi} \Gamma\left(\frac{-2}{a}\right)}{\Gamma\left(\frac{3}{2}+\frac{2}{a}\right)}
$$

Finally, in (5), $P_{e}$ is calculated using numerical integration. Because $Z$ is an $\alpha$-stable random variable, we do not have an expression of its probability density function. To solve this difficulty and obtain $\mathbb{P}\left(Z<-x \mid a_{0}^{(0)}=0, Z_{n}\right)$, we simulate the random variable $Z$ (see for example [24, p. 27-29]) and use a non parametric estimate of its probability density function. In Figure 3 we represent several situations (different values of the attenuation coefficient $a$, the mean number of users per unit area $\lambda$ and the size of the considered zone $R$ ). We show a good fit between the semi-analytical BER and the simulated system for the rectangular pulse. However, this fit is not always perfect and some errors can sometimes be noticed. In such cases we adjust the dispersion of the distribution and obtain an accurate fit between the curves. Similar behavior are obtained with other pulse shapes but the dispersion has to be estimated because we do not have an analytical expression for $F$. We do not propose in this paper discussion about the parameter estimation of a stable law but several works on the subjects exist (see for instance recent works in [27]).

For a rectangular shape, the MAI distribution's parameters are analytically derived and the good fit validates the theoretical study. Similar results are obtained if we increase the number of repetitions. The impact of strong interferers, which generate a heavy tailed distribution, is well captured by the $\alpha$-stable distributions when other distributions with finite second order moments generally fail to do so. For instance, Generalized Gaussian distributions are well fitted for time hopping [28] but does not represent as well the ad hoc configuration [29].

The simulated BER with a near field assumption of $10 \mathrm{~cm}$ is also depicted in Figure 3 (top-right). This value is much larger than the true near field area if a $60 \mathrm{GHz}$ transmission is considered and the impact is very small although the useful link is only ten times longer. 

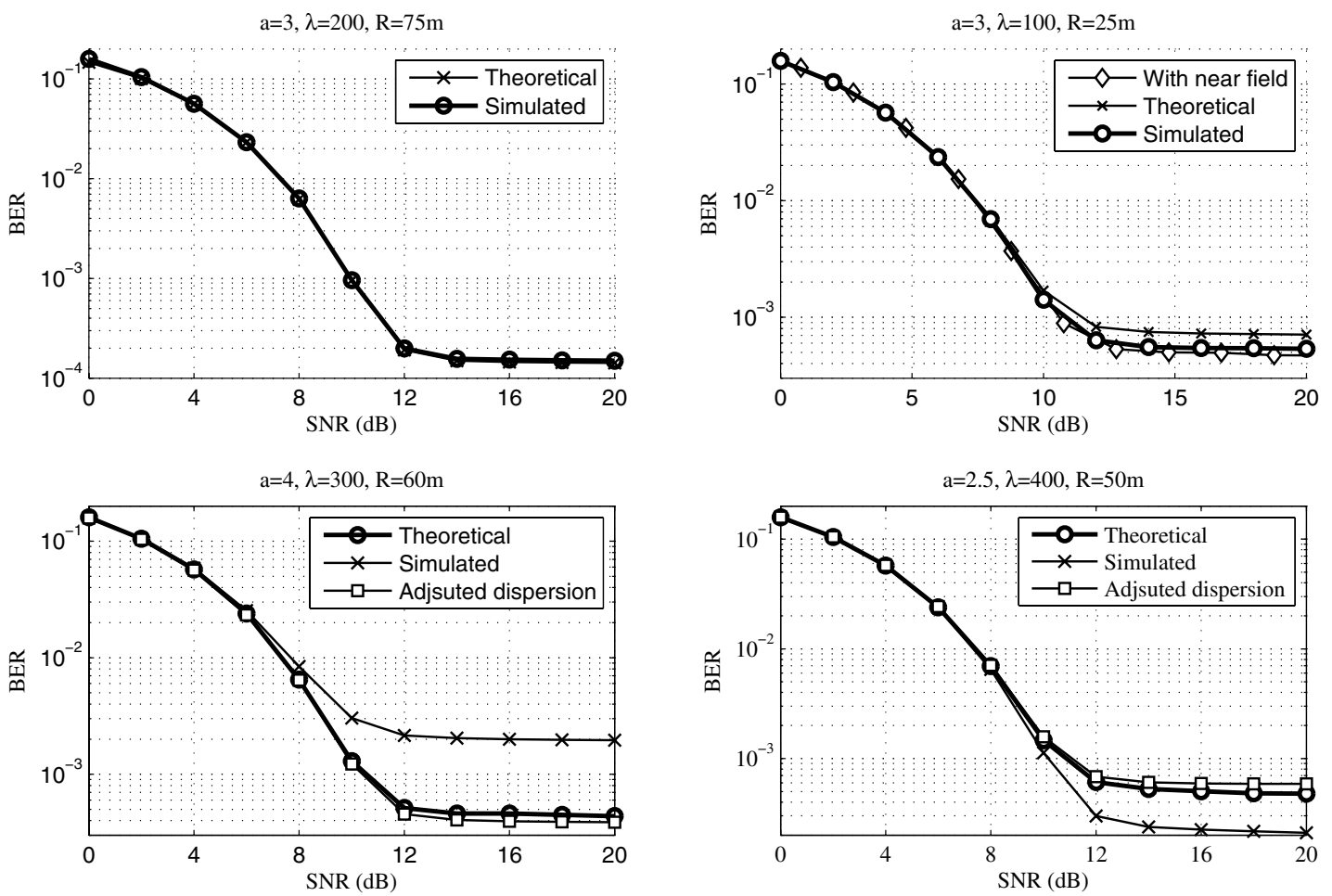

Fig. 3. Comparison between semi-analytical and simulated BER in different situations with a rectangular pulse. The signal to noise ratio is the ratio between the useful signal power and the thermal noise power at the receiver, not including the multiple access noise.

\section{A NON LINEAR RECEIVER IN MULTIPATH CONDITIONS}

\section{A. Receiver strategy.}

Classical receivers consider that the received signal is mixed with a Gaussian noise and are not optimal in our situation. In IR-UWB system, Fiorina [28] models the MAI with a generalized Gaussian distribution to derive the maximum likelihood matched filter. Beaulieu [30] assumes that the signal is immersed in a mixture of Laplacian and Gaussian noise. It outperforms the receiver based on the Gaussian assumption. Considering the impulsive nature of the MAI, Erseghe [31] has suggested Gaussian mixtures for the interference to derive the optimal receiver. Performance improves when compared to the Gaussian receiver and the cost of implementation remains weak. This approach is also found for detection when the additive noise is $\alpha$-stable [32]. Tsihrintzis and Nikias in [33] studied the performance of optimal and suboptimal (including the Cauchy) receivers in such a noise. Those receivers are non linear solutions and a linear solution is proposed in [34]. A non parametric rank-based solution is studied in [35] and suboptimal parametric solutions are presented in [36]. Ambike et al. [37] have also tested a hole puncher and a soft limiter receivers in a mixture of Gaussian and $\alpha$-stable noises. All proposed approaches are compromises between performance and complexity and an overview can be found in [26].

We have chosen to test the non linear Cauchy receiver, meaning the receiver obtained when we consider that a Cauchy noise is added to the information bearing signal. This receiver was found to perform closely to the optimum receiver for a wide range of characteristic exponent $\alpha$ [33]. It was also shown by Friedmann et al. in [38] that the maximum likelihood estimator of a deterministic signal derived for a Cauchy noise achieves good performance when the noise is symmetric $\alpha$-stable, even when $\alpha$ is not equal to one. The definition of the optimal receiver is however a difficult task and is not included in the scope of this paper. Indeed, the noise is not an $\alpha$-stable noise but a mixture of three different noises: the $\alpha$ stable multiple access interference, the Gaussian thermal noise and the multipath interference. The resulting noise distribution is then very difficult to obtain.

Let (as defined in section II) $a_{0}^{(0)}$ be the source bit from the desired user. We consider that the correlation function $\psi_{0}$ in (6) is equal to 1 if $\Delta_{0}=0$ (meaning a perfect synchronization and $a_{0}^{(0)}=0$ ) and to -1 if $\Delta_{0}=\epsilon$ (meaning a perfect synchronization and $a_{0}^{(0)}=1$ ). For the desired user, the received samples are then, after the correlation receiver: $x_{0}^{(0)}(j)=d_{u}^{-\frac{a}{2}}(-1)^{a_{0}^{(0)}}+n_{\alpha}(j)$ where index $j$ indicates the repetitions $\left(j=1, \ldots, N_{S}\right)$. Sequence $\left\{n_{\alpha}(j)\right\}_{j=1, \ldots, N_{S}}$ is a realization of $N_{S}$ independent, identically distributed symmetric $\alpha$-stable random variables. Distance $d_{u}$ is the useful link length. The optimum (in the maximum likelihood sense) test statistic $\Lambda$ is:

$$
\Lambda=\sum_{j=1}^{N_{S}} \log \left\{\frac{f_{\alpha}\left[x_{0}^{(0)}(j)-d_{u}^{-\frac{a}{2}}\right]}{f_{\alpha}\left[x_{0}^{(0)}(j)+d_{u}^{-\frac{a}{2}}\right]}\right\},
$$

where $f_{\alpha}(u)$ is the probability density function of $n_{\alpha}$ and the sign of $\Lambda$ gives the estimated signal. As already mentioned, 
the main difficulty is that $f_{\alpha}(u)$ has not an explicit form for most of $\alpha$ values. Fortunately, $f_{\alpha}$ is known for $\alpha=2$, the Gaussian case, and $\alpha=1$ where $n_{\alpha}$ is a Cauchy random variable $\left(f_{\alpha}(u)=\sigma /\left(\pi\left[u^{2}+\sigma^{2}\right]\right)\right)$. Our objective is to propose a receiver able to cope with impulsive noise. The Cauchy receiver, resulting from our analysis on the MAI, is then the first solution that comes to mind. By Cauchy receiver, we mean the receiver that employs $\Lambda$ derived from (11) under the assumption that $\alpha=1$. The Gaussian receiver uses the assumption that $\alpha=2$.

We have also implemented receivers based on the metric induced by the covariation norm $\|\cdot\|_{\alpha}$ (see for instance [25, p. 95]). From a practical point of view, this norm is linked to the $L^{p}$ norm, with $p<\alpha$, by the equality:

$$
\|X\|_{\alpha}=C_{\alpha}(p)\left(E|X|^{p}\right)^{\frac{1}{p}}
$$

the constant $C_{\alpha}(p)$ depends only on $\alpha$ and $p$ and is given by (see for instance [24, p. 32]):

$$
C_{\alpha}(p)=\left(\frac{\alpha \sqrt{\pi} \Gamma\left(-\frac{p}{2}\right)}{2^{p+1} \Gamma\left(\frac{1+p}{2}\right) \Gamma\left(-\frac{p}{\alpha}\right)}\right)^{\frac{1}{p}} .
$$

This suggests an empirical estimation of this metric by:

$$
\|X-Y\|_{\alpha}=C_{\alpha}(p)\left(\frac{1}{N_{S}} \sum_{j=1}^{N_{S}}\left|x_{j}-y_{j}\right|^{p}\right)^{\frac{1}{p}}
$$

where $p<\alpha$. Two ideas lie behind this metric. First, if Euclidean distance is a logical estimation of standard deviation when Gaussian noise is considered, the proposed solution is adapted to $\mathrm{S} \alpha \mathrm{S}$ distributions since it estimates its scale parameter. Choosing an adapted distance seems then rather logical. The second aspect is that we do not want to give too much weight for the decision to large values because they are mixed with an important noise sample. The Euclidean distance is poorly adapted in that sense and we prefer using the covariation norm. Beaulieu et al. proposed a similar solution in [18] that is an optimal solution for generalized Gaussian interference. We however did not use their estimation procedure for $p$ which is not consistent with our approach. It is based on second and higher order moments which are not defined for stable distributions.

Finally, to get an idea of an optimal solution, we have implemented a genie-aided receiver: in the simulation we extract the exact noise samples and use a kernel type non parametric estimation of the noise distribution (a Gaussian kernel is considered). We then use this distribution to calculate the log-likelihood function. This distribution is calculated on each packet that we have taken rather short (50 source bits repeated 4 times) to avoid too much computation complexity). In future works, we will focus on solutions using some approximate methods of the optimal receiver performance, which represents a challenging problem owing to the $\alpha$-stable nature of the MAI interference process.

\section{B. Simulations}

We have considered two scenarios. The first one uses a Gaussian channel so that only Gaussian noise and multiple access interference play a role. The second one includes an important multipath impact. Figure 4 presents the BER as a function of the mean number of users $\lambda$. On the left graph, with no multipath, we clearly see the improvement brought by the Cauchy receiver, the number of errors being reduced by a factor 10. The proposed metric is also an attractive solution when $p$ takes small values. For $p=0.5$, the proposed metric gives similar results to the Cauchy receiver. The genie-aided receiver exhibits of course better performance. It is not clear how close to this optimal curve we can get.

We have also simulated an ad hoc network situation as described in section II but with a multipath channel. We have chosen the residential model proposed by the IEEE $802.15 .4 \mathrm{a}$ [39], [40]. The useful link is in Line Of Sight (LOS) while the interfering links can be either LOS or non LOS with probability 0.5 . The global power attenuation is $d^{-a}$ and an additional shadowing factor of 0.5 is used for non LOS users. Each object uses the same transmit power. The improvement due to the Cauchy receiver is reduced but still significant. The conclusion on the use of the proposed metric are similar to the previous case. The optimal solution offers a further gain but the much more difficult transmission conditions make the benefit smaller.

An appropriate solution for an indoor ad hoc networks, eventually at $60 \mathrm{GHz}$, would be to use directive antennas and links with a LOS path. The resulting conditions would then be in between the two considered scenarios, the importance of multipath depending on the antenna directivity and the environment. The benefits resulting from the proposed metric or the Cauchy receiver would then be significant. Taking the impulsive nature of the interference into account is important and the stable model can certainly bring an accurate general mathematical framework [7].

\section{CONCLUSION}

In this paper we present a study of multiple access interference in ad hoc networks based on TH-PPM-UWB. We propose a model based on $\alpha$-stable process. Those distributions are heavy tailed and perfectly capture the interference nature: strong interferers (close to the receiver) are the ones that limit the system performance, but they are rare. Moreover, the time hopping approach further limits their influence. Gaussian distributions or, in general, random variables with finite second order moments do not allow a good representation of these events. On the other hand, the proposed $\alpha$-stable random variables are very well suited for modeling this impulsive noise.

For a single path channel and neglecting the near field, we demonstrate that multiple access interference is a symmetric $\alpha$-stable random variable and derive an analytical expression of the two parameters defining the distribution. The limitation of the received power in the near field has no real impact on our modeling. The multipath do not change the MAI nature but a new evaluation of the parameters is necessary.

Because we have no exact expression of the probability density functions of $\alpha$-stable random variables, an optimal receiver can not be easily implemented. We test receivers for $\alpha=1$ (Cauchy receiver) and $\alpha=2$ (Gaussian receiver). 

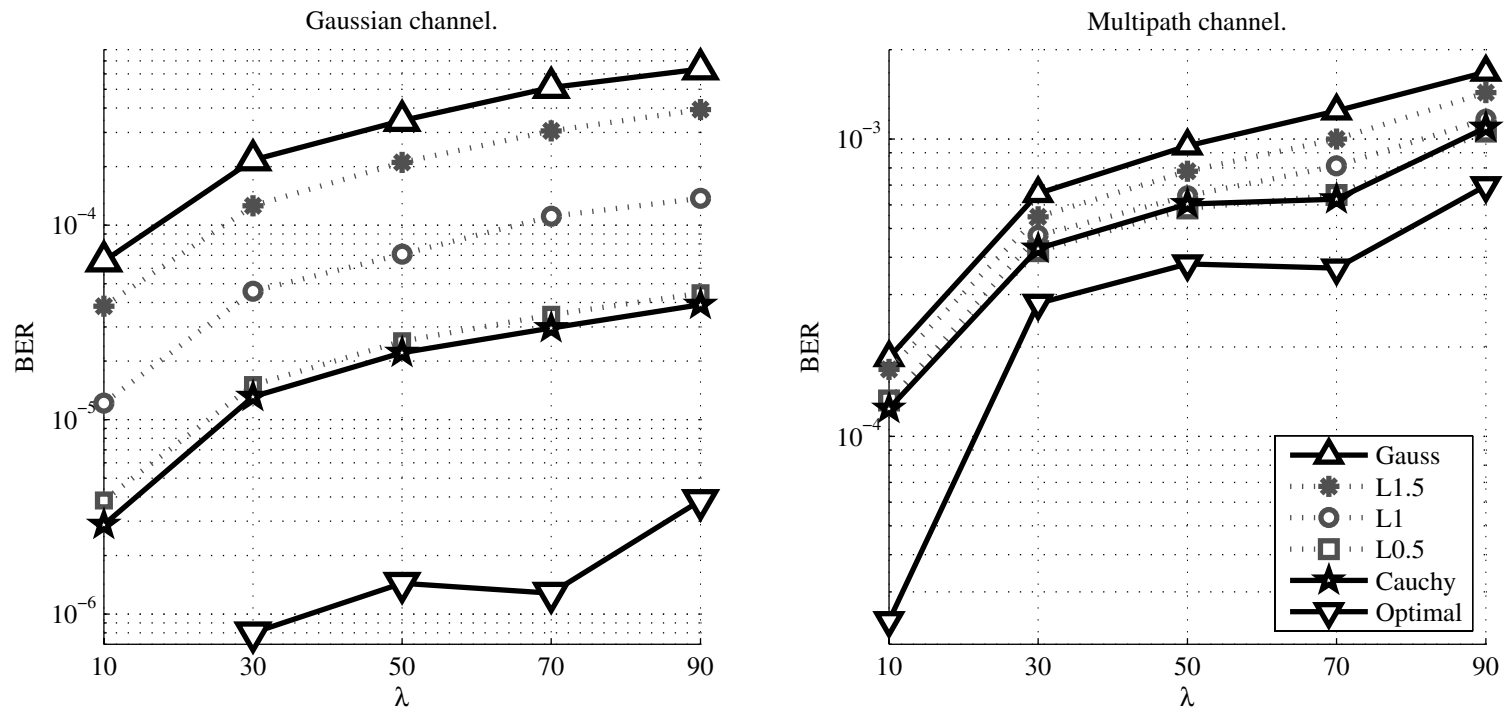

Fig. 4. BER as a function of the mean number of users for different receivers and two different scenarios. The SNR per pulse after the correlation receiver is $10 \mathrm{~dB}$. The radius of the area is $R=30 \mathrm{~m}$.

We also propose a receiver based on a metric inspired by the covariation norm, well adapted to $\alpha$-stable processes. We notice a clear improvement of performance with the two proposed strategies, which confirms the heavy tailed nature of the MAI.

Further works have to be done to better integrate the results of this paper in ad hoc networks. Better receivers can certainly be implemented to account for the mixture of different noises and optimal bounds have to be found. Besides, some estimation difficulties will be encountered to determine the distribution parameters. Finally, some works on the channel modeling is also necessary because we can not consider identical models for the desired link, short with a line of sight, and the interfering one, long and possibly without a line of sight.

\section{APPENDIX A $\alpha$-STABLES DISTRIBUTIONS}

The $\alpha$-stable processes and variables are a direct generalization of Gaussians' and share many of their familiar properties (see [24], [25] for more details on $\alpha$-stable processes and signal processing). In particular:

- The convolution stability property, which means that the convolution of two stable distributions is also stable. In other words the sum of two independent stable random variables is also a stable one.

- The central limit theorem, which means that every stable random variables may be expressed as a limit, in distribution, of a normalized sum of independent and identically distributed random variables.

Another aspect that makes the importance of these distributions is that they are parametric. Indeed they are fully described by four parameters:

- $\alpha$ is called the characteristic exponent $(0<\alpha \leq 2)$ : it measures the thickness of the tail of the distribution. Thus larger is the value of $\alpha$, less likely it is to observe values which are far from the central location.

- $\mu$ is the location parameter $(-\infty<\mu<\infty)$ : in an observed sample most of the observations are concentrated about its value. It corresponds to the mean for $1<\alpha \leq 2$ and to the median for $0<\alpha \leq 1$

- $\sigma$ is the dispersion parameter $(\sigma>0)$ : it has a similar role as the standard deviation in the case of a Gaussian distribution.

- $\beta$ is the index of symmetry $(-1 \leq \beta \leq 1)$ which characterizes the dissymmetry of the density function about its central location. When $\beta=1$ we say that the distribution is totally skewed to the right; it is symmetric if $\beta=0$.

In this paper we consider symmetric $\alpha$-stable random variables, which means that $\beta=\mu=0$.

Since their discovery by Paul Levy in 1925, a great amount of knowledge has been accumulated about the theoretical properties of these probability distributions. They have been found to provide useful models in various application fields, especially phenomena with large fluctuations and high variability that are not compatible with the Gaussian models. Except the Gaussian, the Cauchy and the Levy distributions, which are special cases of the stable class, there is no exact expression of the probability density function of an $\alpha$-stable distribution. However its characteristic function is given for $\alpha \neq 1$ by:

$$
\Phi(\theta)=\exp \left\{-\sigma^{\alpha}|\theta|^{\alpha}\left(1-i \beta \operatorname{sign}(\theta) \tan \frac{\pi \alpha}{2}\right)+i \mu \theta\right\}
$$

and for $\alpha=1$ by:

$$
\Phi(\theta)=\exp \left\{-\sigma|\theta|\left(1+i \beta \frac{2}{\pi} \operatorname{sign}(\theta) \ln |\theta|\right)+i \mu \theta\right\}
$$

where

$$
\operatorname{sign}(\theta)=\left\{\begin{array}{cl}
1 & \text { if } \quad \theta>0 \\
0 & \text { if } \quad \theta=0 \\
-1 & \text { if } \quad \theta<0
\end{array} .\right.
$$




\section{APPENDIX B}

\section{THE MAI LOG-CHARACTERISTIC FUNCTION}

We assume that $\bar{N}$ (the mean number of interfering links in the circle $C$ of radius $R$ ) follows a Poisson law of parameter $\lambda \pi R^{2} . \lambda$ is the expected number of interferer per unit area which is linked to the density of the network. We use the demonstration proposed by Sousa [4] determining the log characteristic function of the MAI.

Knowing the number of active links $N$ in $C$, the number of interfering pulses $\kappa_{R}$ is a binomial law $B(N, q) . q$ is the probability that one interfering pulse falls into the integration interval at the receiver. The number of interfering pulses is a Poisson law of parameter $\lambda \pi R^{2} q$.

$$
\mathbb{P}\left(\kappa_{R}=i\right)=\frac{e^{-\lambda q \pi R^{2}}\left(\lambda q \pi R^{2}\right)^{i}}{i !} .
$$

Using (6) and (18), the MAI characteristic function $\phi_{Z}(\omega)=\mathbb{E}\left[e^{j w Z}\right]$ can be written:

$$
\phi_{Z}(\omega)=\mathbb{E}\left[e^{j \omega\left(\sum_{i=1}^{\kappa} \gamma_{i} \psi_{i}\right)}\right],
$$

where $\kappa$ is the number of interferers in the circle with radius $+\infty$ (to model our system, we make $R$ tends towards infinity). We have $\mathbb{P}(\kappa=l)=\lim _{R \rightarrow+\infty} \mathbb{P}\left(\kappa_{R}=l\right)$. Since $\psi_{i}$ and $\gamma_{i}$ are independent, identically distributed and independent of $\kappa$, we can write:

$$
\begin{aligned}
\phi_{Z}(\omega) & =\sum_{l=0}^{+\infty} \mathbb{P}(\kappa=l) \cdot\left(\mathbb{E}\left[e^{j \omega(\gamma \psi)}\right]\right)^{l} \\
& =\sum_{l=0}^{+\infty} \lim _{R \rightarrow+\infty}\left(\frac{e^{-\lambda \pi R^{2} q} \cdot\left(\lambda \pi R^{2} q\right)^{l}}{l !}\right) \cdot\left(\mathbb{E}\left[e^{j \omega \gamma \psi}\right]\right)^{l} \\
& =\lim _{R \rightarrow+\infty} e^{-\lambda \pi R^{2} q} e^{\lambda \pi R^{2} q \mathbb{E}\left[e^{j \omega \gamma \psi}\right]} .
\end{aligned}
$$

Let $\omega>0$; we take the log-characteristic function:

$$
\begin{aligned}
\varphi_{Z}(\omega) & =\log \left(\phi_{Z}(\omega)\right) \\
& =\lim _{R \rightarrow+\infty} \lambda q \pi R^{2}\left(\mathbb{E}\left[e^{j \omega \gamma \psi}\right]-1\right) .
\end{aligned}
$$

The expectation is taken over the two random variables $\gamma$ and $\psi$ and can be calculated using (7):

$$
\begin{aligned}
\mathbb{E}\left[e^{j \omega \gamma \psi}\right] & =\int_{R^{-\frac{a}{2}}}^{+\infty} \mathbb{E}\left[e^{j \omega \gamma \psi} \mid \gamma=x\right] f_{\gamma}(x) d x \\
& =\int_{R^{-\frac{a}{2}}}^{+\infty} \phi_{\psi}(\omega x) \frac{4 x^{-\frac{4}{a}-1}}{a R^{2}} d x .
\end{aligned}
$$

Integrating (22) by parts, using it in (21) and making a change of variables $(u=\omega x)$ we obtain:

$$
\begin{aligned}
\varphi_{Z}(\omega)= & \lim _{R \rightarrow+\infty} \lambda q \pi R^{2}\left\{\phi_{\psi}\left(\omega R^{-\frac{a}{2}}\right)\right. \\
& \left.+\frac{1}{R^{2}} \int_{\omega R^{-\frac{a}{2}}}^{+\infty} \frac{d \phi_{\psi}}{d u}(u)\left(\frac{u}{\omega}\right)^{-\frac{4}{a}} d u-1\right\}
\end{aligned}
$$

Since $\psi$ is a random variable with mean 0 and a finite variance $\sigma_{\psi}^{2}$, we can give the Taylor expansion of $\phi_{\psi}(x)$, for $x$ near 0 , to get the approximations:

$$
\begin{array}{r}
\phi_{\psi}(x)=1-\frac{\sigma_{\psi}^{2}}{2} x^{2}+o\left(x^{2}\right) \\
\text { and } \quad \frac{d \phi_{\psi}}{d x}(x)=-\sigma_{\psi}^{2} x+o(x) .
\end{array}
$$

Consequently,

$$
\lim _{R \rightarrow+\infty} \lambda q \pi R^{2}\left(\phi_{\psi}\left(\omega R^{-\frac{a}{2}}\right)-1\right)=0
$$

and the approximation of $\frac{d \phi_{\psi}}{d x}$ allows to justify that only the other term in (23) remains. We can further develop (23):

$$
\begin{aligned}
\varphi_{z}(\omega) & =\lambda q \pi \omega^{\frac{4}{a}} \int_{0}^{+\infty} \frac{d \phi_{\psi}}{d u}(u) u^{-\frac{4}{a}} d u \\
& =\lambda q \pi|\omega|^{\frac{4}{a}} \int_{0}^{+\infty} \frac{d \phi_{\psi}}{d u}(u) u^{-\frac{4}{a}} d u .
\end{aligned}
$$

Since $\psi$ is a real centered symmetric random variable, the characteristic function $\phi_{\psi}$ is an even function. If now $\omega$ is negative then by a similar calculation, we find that:

$$
\begin{aligned}
\varphi_{Z}(\omega) & =\lambda q \pi(-\omega)^{\frac{4}{a}} \int_{0}^{+\infty} \frac{d \phi_{\psi}}{d u}(u) u^{-\frac{4}{a}} d u \\
& =\lambda q \pi|\omega|^{\frac{4}{a}} \int_{0}^{+\infty} \frac{d \phi_{\psi}}{d u}(u) u^{-\frac{4}{a}} d u .
\end{aligned}
$$

Finally the log-characteristic function of the random variable $Z$ is given by equation (9) that we recall here:

$$
\begin{aligned}
\varphi_{Z}(\omega) & =\lambda q \pi|\omega|^{\frac{4}{a}} \int_{0}^{+\infty} \frac{d \phi_{\psi}}{d u}(u) u^{-\frac{4}{a}} d u \\
& =\lambda q \pi|\omega|^{\frac{4}{a}} F,
\end{aligned}
$$

where $F$ is independent of $\omega$ and given by:

$$
F=\int_{0}^{+\infty} \frac{d \phi_{\psi}}{d u}(u) u^{-\frac{4}{a}} d u .
$$

This gives the characteristic function of the random variable $Z$ :

$$
\phi_{Z}(\omega)=e^{-\sigma|\omega|^{\alpha}} .
$$

Then, the MAI distribution is an $\alpha$-stable distribution with $\alpha=\frac{4}{a}$ and $\sigma=-\lambda q \pi F$.

\section{ACKNOWLEDGMENT}

This work was supported by the ERDF (European Regional Development Fund) and by the Nord-Pas-de-Calais Region (France).

\section{REFERENCES}

[1] M. Win and R. Scholtz, "Ultra-wide bandwidth time-hopping spreadspectrum impulse radio for wireless multiple-access communications," IEEE Trans. Commun., vol. 48, no. 4, pp. 679-689, Apr. 2000.

[2] A. Forouzan, M. Nasiri-Kenari, and J. Salehi, "Performance analysis of time hopping spread-spectrum multiple access system: uncoded and coded schemes," IEEE Trans. Wireless Commun., vol. 1, no. 4, pp. 671681, Oct. 2002.

[3] G. Durisi and S.Benedetto, "Performance evaluation of TH-PPM UWB systems in the presence of multiuser interference," IEEE Commun. Lett., vol. 7, no. 5, pp. 224-226, May 2003.

[4] E. Sousa, "Performance of a spread spectrum packet radio network in a poisson field of interferers," IEEE Trans. Inf. Theory, vol. 38, no. 6, pp. 1743-1754, Nov. 1992.

[5] J. Ilow and D. Hatzinakos, "Impulsive noise modeling with stable distributions fading environments," in 8th IEEE Signal Process. Workshop Statistical Signal Array Process., June 1996, pp. 140-143.

[6] P. Pinto, C.-C. Chong, A. Giorgetti, M. Chiani, and M. Win, "Narrowband communication in a poisson field of ultrawideband iinterferers," in The IEEE 2006 International Conf. Ultra-Wideband (ICUWB), Sept. 2006, pp. 387-392. 
[7] M. Win, P. Pinto, and L. Shepp, "A mathematical theory of network interference and its applications," in Proc. IEEE, vol. 97, no. 2, pp. 205-230, Feb. 2009

[8] G. Durisi and G. Romano, "On the validity of gaussian approximation to characterize the multiuser capacity of uwb th-ppm," IEEE Conf. Ultra Wideband Syst. Technol., pp. 20-23, May 2002.

[9] C. Martret, A.L.Deleuze, and P. Ciblat, "Optimal time-hopping codes for multi-user interference mitigation in ultra-wide bandwidth impulse radio," IEEE Trans. Wireless Commun., vol. 5, no. 6, pp. 1516-1525, June 2006.

[10] G. Durgin, Space-Time Wireless Channels. Prentice Hall PTR, Oct. 2002.

[11] B. $\mathrm{Hu}$ and N. C. Beaulieu, "Accurate evaluation of multiple-access performance in TH-PPM and TH-BPSK UWB systems," IEEE Trans. Commun., vol. 52, pp. 1758-1766, Oct. 2004.

[12] G. Giancola and M.-G. D. Benedetto, "A novel approach for estimating multi-user interference in impulse radio uwb networks: The pulse collision model," EURASIP Signal Process. J., Special Issue Signal Process. UWB Commun., vol. 86, pp. 2185-2197, 2006.

[13] N. Deparis, A. Bendjabballah, A. Boe, M. Fryziel, C. Loyez, L. Clavier, N. Rolland, and P. Rolland, "Transposition of a baseband uwb signal at $60 \mathrm{GHz}$ for high data rate indoor WLAN," IEEE Microwave Wireless Compon. Lett., vol. 15, no. 10, pp. 609-611, Oct. 2005.

[14] A. Boé, M. Fryziel, N. Deparis, C. Loyez, L. Clavier, N. Rolland, and P. A. Rolland, "Smart antenna based on low-temperature MEMS switches and quasi-Yagi antennas for WPAN," in 4th ESA Workshop Millimetre Wave Techn. Appl., Feb. 2006.

[15] A. Bendjaballah, L. Clavier, N. Rolland, and P. A. Rolland, "Multihop channel model in uwb ad hoc networks at $60 \mathrm{ghz}, "$ in First European Conf. Antennas Propagation (EuCAP 2006), Nice, France, Nov. 2006.

[16] F. Baccelli, B. Blaszczyszyn, and P. Muhlethaler, "An aloha protocol for multihop mobile wireless networks," IEEE Trans. Inf. Theory, vol. 52, no. 2, pp. 421-436, Feb. 2006.

[17] J. Zhang, P. Orlik, Z. Sahinoglu, A. Molisch, and P. Kinney, "UWB systems for wireless sensor networks," Proc. IEEE, vol. 97, no. 2, pp. 313-331, Feb. 2009

[18] N. Beaulieu, H. Shao, and J. Fiorina, "P-order metric uwb receiver structures with superior performance," IEEE Trans. Commun., vol. 56, no. 10 , pp. 1666-1676, Oct. 2008.

[19] H. Ghannudi, L. Clavier, A. Bendjaballah, A. Boé, and P. Rolland, "Performance of IR-UWB at $60 \mathrm{GHz}$ for ad hoc networks with directive antennas," in IEEE 2006 International Conf. Ultra-Wideband (ICUWB), Sept. 2006, pp. 149-154.

[20] P. Smulders, "Exploiting the $60 \mathrm{GHz}$ band for local wireless multimedia access: Prospects and future direction," IEEE Commun. Mag., vol. 40, no. 1, pp. 140-147, Jan. 2002.

[21] A. Bendjaballah, H. E. Ghannudi, N. Deparis, A. Boé, and L. Clavier, "Channel model and performance of ad hoc networks based on IR-UWB at $60 \mathrm{GHz}, "$ in 4th ESA Workshop Millimetre Wave Techn. Appl., Feb. 2006.

[22] M. Fryziel, C. Loyez, L. Clavier, and N. Rolland, "Path loss model of the $60 \mathrm{GHz}$ radio channel," Microwave Optical Technol. Lett., vol. 34, no. 3, pp. 158-162, Aug. 2002.

[23] J. Fiorina and W. Hachem, "On the asymptotic distribution of the correlation receiver output for time-hopped uwb signals," IEEE Trans. Signal Process., vol. 54, no. 7, pp. 2529-2545, July 2006.

[24] S. M. Nikias C. L., Signal Processing with $\alpha$-stable Distributions and Applications, W. inter science, Ed. J.Wiley, 1995.

[25] G. Samorodnitsky and M. Taqqu, Stable Non-Gaussian Random Processes : Stochastic Models with Infinite Variance. Chapmann and Hall, 1994.

[26] N. Beaulieu and D. Young, "Designing time-hopping ultrawide bandwidth receivers for multiuser interference environments," Proc. IEEE, vol. 97, no. 2, pp. 255-284, Feb. 2009.

[27] P. Besbeas and B. Morgan, "Improved estimation of the stable laws," Statistics Comput., vol. 18, no. 2, pp. 219-231, 2008.

[28] J. Fiorina, "A simple IR-UWB receiver adapted to Multi-User Interferences," in IEEE Global Telecommun. Conf., GLOBECOM 2006, Nov. 2006, pp. 1-4.

[29] H. E. Ghannudi, L. Clavier, and P. Rolland, "Modeling multiple access interference in ad hoc networks based on IR-UWB signals up-converted to $60 \mathrm{GHz}$," in European Conf. Wireless Technol., Munich, Germany, Oct. 2007, pp. 106-109.

[30] N. Beaulieu and S. Niranjayan, "New UWB receiver designs based on a Gaussian-Laplacian noise-plus-MAI model," in International Conf. Commun., ICC 2007, June 2007, pp. 4128-4133.
[31] T. Erseghe, "A low-complexity impulse radio receiver based upon Gaussian mixtures," in IEEE Int. Conf. Commun., ICC 2007, June 2007, pp. 4311-4316.

[32] E. Kuruoglu, W. Fitzgerald, and P. Rayner, "Near optimal detection of signals in impulsive noise modeled with a symmetric $\alpha$-stable distribution," IEEE Commun. Lett., vol. 2, no. 10, pp. 282-284, Oct. 1998.

[33] G. Tsihrintzis, C. Nikias, and M. Shao, "Performance of optimum and suboptimum receivers in the presence of impulsive noise modeled as an alpha-stable process," IEEE Trans. Commun., vol. 43, no. 2, pp. 904914, Feb. 1995

[34] D. Johnson, "Optimal linear detectors for additive noise channels," IEEE Trans. Signal Process., vol. 44, no. 12, pp. 3079-3084, Dec. 1996.

[35] C. Brown and A. Zoubir, "A nonparametric approach to signal detection in impulsive interference," IEEE Trans. Signal Process., vol. 48, no. 9 , pp. 2665-2669, 2000 .

[36] S. Zozor, J. Brossier, and P. Amblard, "A parametric approach to suboptimal signal detection in $\alpha$-stable noise," IEEE Trans. Signal Process., vol. 54, no. 12, pp. 4497-4509, Dec. 2006.

[37] S. Ambike, J. Ilow, and D. Hatzinakos, "Detection for binary transmission in a mixture of gaussian noise and impulsive noise modeled as an alpha-stable process," IEEE Signal Process. Lett., vol. 1, no. 3, pp. 55-57, Mar. 1994

[38] J. Friedmann, H. Messer, and J. Cardoso, "Robust parameter estimation of a deterministic signal in impulsive noise," IEEE Trans. Signal Process., vol. 48, no. 4, pp. 935-942, Apr. 2000.

[39] A. Molisch, J. Foerster, and M. Pendergrass, "Channel models for ultrawideband personal area networks," IEEE Wireless Commun. Mag., vol. 10 , no. 6 , pp. 14-21, Dec. 2003.

[40] A. F. Molisch, D. Cassioli, C. Chong, S. Emami, A. Fort, B. Kannan, J. Karedal, J. Kunisch, H. Schantz, K. Siwiak, and M. Win, "A comprehensive standardized model for ultrawideband propagation channels," IEEE Trans. Antennas Propagat., vol. 54, no. 11, part 1, pp. 3151-3166, Nov. 2006.

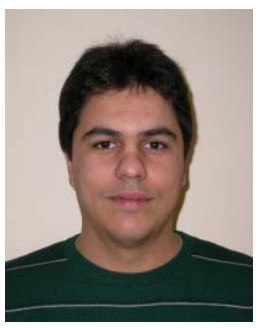

Hamza El Ghannudi was born in Manosque, France, on December 19, 1978. He received a B.S. degree in Electronic and Telecommunication engineering from Al-Fateh University, Tripoli, Libya, in 2002, the M.S. degree in Microwave and Electronic engineering from University of Science and Technology of Lille, France, in 2004, the Ph.D. degree in Microwave and Electronic engineering from the "Institut d'Electronique et de Microélectronique et de Nanotechnologie (IEMN)", Lille, France, in 2007. During his Ph.D, he worked on the performance of Impulse Radio Ultra Wide Band system up converted to the $60 \mathrm{GHz}$ band for high rate ad hoc network. In April 2008 he joined the Department of Electronic and Information Engineering (DIEI) of the University of Perugia (Italy) as Post-Doc researcher and RF Microtech as R\&D Engineer. His research activities include the electromagnetic modeling and design of RF MEMS and packaging, design and simulation of reflectarrays antenna and positioning systems in indoor environment.

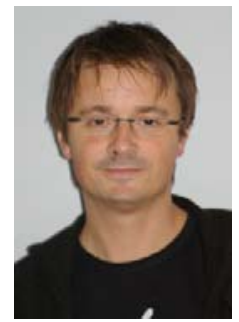

Laurent Clavier received the M.S.degree from ENSEEIHT in Toulouse, France, in 1993. In 1997 he received his $\mathrm{PhD}$ degree in signal processing in TELECOM Bretagne in Brest, France. He is currently an associate professor in TELECOM Lille 1 , an engineering school member of the TELECOM Institute in France. He is with the Institute of Electronics, Microelectronics and Nanotechnology (IEMN - UMR CNRS 8520) and with the Research Institute on hardware and software components for advanced information and communication (IRCICA - FR CNRS 3024) in Lille, France. His main research areas are about statistical channel and interference modeling and on the optimisation of the physical layer for ad hoc and sensor networks. 


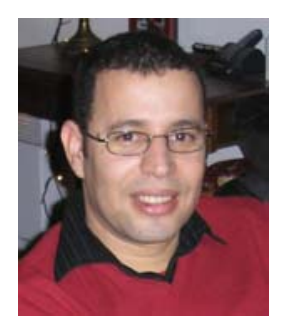

Nourddine Azzaoui received the Maîtrise diploma in applied mathematics in 1999, and the DESA degree of statistics and applied probability in 2002 from Fez University in Morocco. He then obtained the Ph.D. degree in 2006 from Burgundy University in Dijon France. From 2006 to 2008, he was an assistant professor(ATER) respectively at the Burgundy mathematics institute and at the Paul Painlevé Laboratory of mathematics in Lille France; he was in the statistics and probability team. Since September 2009, he is Postdoc researcher at the Systems Modeling and Dependability Laboratory at Troyes University of Technology.

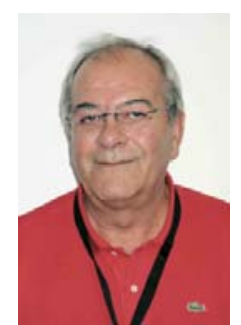

Paul-Alain Rolland received a Engineer degree in electronics in 1971, a $\mathrm{PhD}$ degree in 1973 and thèse d'État degree in 1978. He is currently Professor at the University of LILLE. He is head of the microwave department of IEMN, responsible of the Circuit System and Microwave Application group of IEMN and develops research in the field of advanced communication systems for SOC and sensors networks in millimeter wave range. $\mathrm{He}$ is also head of IRCICA.

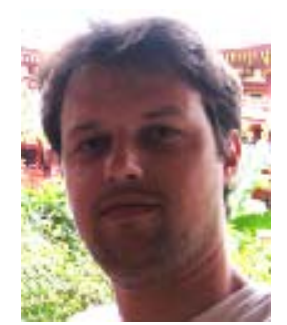

François Septier received the Engineer degree in electrical engineering and signal processing in 2004 from TELECOM Lille 1, France, and the M.S. degree in digital communications and a Ph.D. in Electrical Engineering both from the University of Valenciennes, France, in 2004 and 2008 respectively. From 2008 to 2009, he was a Research Associate in the Signal Processing and Communications Laboratory, Cambridge University Engineering Department, UK. Currently, he is an Assistant Professor at TELECOM Lille 1, France, and a member of the LAGIS laboratory (UMR CNRS 8146). His research focuses on Monte-Carlo statistical methods for digital communications, multitarget tracking and source term estimation. 